\title{
PHYDIS - PC-geStütZTe STANDORTBeSTIMMUNG IN EUROPA
}

\section{Karl-Heinz Brockmann, Forschungsinstitut für Rationalisierung an der RWTH Aachen}

Vor dem Hintergrund der Vollendung des EG-Binnenmarktes und der damit verbundenen distributionslogistischen Konsequenzen stellt sich für viele europaweit operierende Unternehmen das Problem der Neugestaltung ihres Warenverteilungssystems.

Die Struktur eines Warenverteilungssystems wird dabei durch die Realisation der folgenden vier Entscheidungsvariablen determiniert:

- Zahl der Distributionsstufen

- Zahl der Läger je Stufe

- Standorte der Läger

- Liefergebiete der Läger.

In der Literatur existiert eine Vielzahl von Veröffentlichungen, die sich mit der Entwicklung von Verfahren zur Strukturierung von Warenverteilungssystemen befassen, wobei die Minimierung der Warenverteilungskosten in aller Regel die Zielfunktion bildet. Dabei existieren zwei Klassen von Lösungsansätzen: Der diskrete Ansatz geht von der Existenz bekannter potentieller Lagerstandorte aus, wohingegen der homogene Ansatz prinzipiell jeden Punkt des untersuchten Gebiets als potentiellen Standort zuläßt.

Im Rahmen dieses Vortrages wurde ein am Forschungsinstitut für Rationalisierung entwickeltes PCgestütztes Verfahren (PHYDIS; PHYsische DIStribution) zur Optimierung von Standorten und Liefergebieten eines Warenverteilungssystems vorgestellt, das von der Modellannahme eines homogenen Territoriums ausgeht. Dabei wird zur Bestimmung optimaler Lagerstandorte auf der Basis fester Liefergebiete ein Iterationsverfahren eingesetzt, das auf der Basis eines Gradientenansatzes entwickelt wurde, während die Zuordnung von Kunden zu Lagerstandorten nach dem Kriterium der geringsten Entfernung erfolgt. Hierbei ist auch die Integration bereits bestehender Läger in ein zu strukturierendes Warenverteilungssystem - gegebenenfalls unter Berücksichtigung von Kapazitätsrestriktionen - möglich. Als Inputdaten benötigt das Verfahren die geographischen Koordinaten der Werks- und Kundenstandorte, die erwarteten bzw. in einem repräsentativen Zeitraum erhobenen Nachfragemengen der Kunden sowie die zur Kostenberechnung erforderlichen Kostensätze. 